$\begin{array}{ll}\text { le portiQue } & \text { Le Portique } \\ \text { Revue de philosophie et de sciences humaines }\end{array}$

$18 \mid 2006$

Heidegger. La pensée à l'heure de la mondialisation

\title{
Avec un v minuscule
}

\section{Fernando Savater}

\section{OpenEdition}

Journals

Édition électronique

URL : https://journals.openedition.org/leportique/832

DOI : 10.4000/leportique.832

ISSN : $1777-5280$

\section{Éditeur}

Association "Les Amis du Portique"

Édition imprimée

Date de publication : 1 septembre 2006

ISSN : 1283-8594

\section{Référence électronique}

Fernando Savater, «Avec un v minuscule », Le Portique [En ligne], 18| 2006, mis en ligne le 15 juin 2009, consulté le 20 février 2023. URL : http://journals.openedition.org/leportique/832 ; DOI : https:// doi.org/10.4000/leportique.832

Ce document a été généré automatiquement le 20 février 2023.

Tous droits réservés 


\title{
Avec un v minuscule
}

\author{
Fernando Savater
}

1 Dans un bistrot proche de Montparnasse, tout le monde fumait, même la caissière. Un scandale. Le garçon, en venant me servir mon Sancerre, me le fit remarquer sur un ton moqueur : «Fumez, mon ami! Vous ne fumez pas? Ah oui, c'est vrai, en Espagne il est interdit de fumer dans les bars. J'ai entendu que bientôt ils interdiront aussi de boire de l'alcool. À ce rythme-là, demain on pourra même plus... » Je lui coupai la parole d'un grognement peu amical, avant de me radoucir, sous l'effet d'une goulée de Sancerre conciliant : «Ça suffit! Écoutez, je viens de voir l'exposition sur la mélancolie au Grand Palais et je ne veux pas mourir d'overdose. " Le garçon ironique haussa les épaules, passa un coup de chiffon sur le comptoir avec un petit air de supériorité et me laissa tranquille.

2 La première définition connue de la mélancolie se trouve chez Hippocrate : "Quand crainte et abattement durent longtemps, cela a un rapport avec la bile noire» (Aphorismes). En fait, l'expérience mélancolique la plus évidente que propose la curieuse exposition organisée par Jean Clair est le succès qu'elle rencontre auprès du public: rien ne provoque davantage un mélange de crainte et d'abattement que de se voir enfermé pendant quelques heures dans un petit espace avec un si grand nombre de nos contemporains. Quant à l'exposition elle-même, on en retire une seule chose claire, à savoir que s'appuyer la tête sur la main constitue un signe extérieur d'ennui depuis au moins vingt-cinq siècles. Les individus pensifs ont vraiment mauvaise réputation... Mais peut-être la littérature, et sans aucun doute la musique, sont-ils davantage adaptés que la peinture pour représenter cet état d'âme doucement noir, mis à part pour certains chefs-d'œuvre isolés comme tel tableau de Caspar David Friedrich ou bien l'admirable New York Movie d'Edward Hopper. Et Goya bien entendu. Son fameux portrait de Jovellanos, exposé au Grand Palais, apporte un supplément de mélancolie « uniquement pour les Espagnols", étant donné qu'il s'agit de notre plus grand homme des Lumières... Or on imagine mal des effigies de Voltaire ou de Diderot pour illustrer cet état d'âme! Que les Lumières espagnoles soient affectées, à un tel degré, par la crainte et l'abattement doit être une spécialité locale, comme le gazpacho. Il est regrettable également que l'exposition ne fasse aucune place au cinéma: le film de Bergman, Les Fraises sauvages, ne représente-t-il pas le meilleur manifeste possible de la mélancolie? 
3 Il est intéressant également de poser la question des remèdes à la mélancolie. L'exposition montre l'un d'entre eux, à savoir la musique (David jouant de la harpe devant Saül), qui reste un antidote ambigu car elle joue en même temps le rôle de stimulant (le tableau de la jeune fille écoutant Schumann). Nous devons ensuite considérer, bien entendu, le vin. Dans son étude classique sur le sujet, Burton rappelle à cet égard le jugement de Rhazès pour qui le vin représente le meilleur traitement de cette maladie : "Celui qui peut trouver de la compagnie pour boire n'a plus besoin d'autres médicaments." Omar Khayyâm partage cette opinion et Avicenne, dans son Canon de la médecine, recommande aux personnes souffrant de mélancolie, non seulement de boire, mais de s'enivrer franchement de temps à autre. Il est difficile de nos jours de trouver des médecins aussi sages. L'austère Sénèque donne le même conseil à l'un de ses correspondants éprouvant de la tristesse, et va jusqu'à parler de "sobria ebrietas", expression qui semble aujourd'hui presque subversive. Au Grand Palais, je n'ai trouvé aucune image de fumeur. Pourtant, le tabac (sans parler de ses grands frères, l'opium et le chanvre) est un médicament réputé contre l'ennui. Dans la peinture hollandaise du Siècle d'or (celle de Pieter Codde par exemple), il est fréquent de rencontrer l'image d'un fumeur de pipe, la tête appuyée sur la main et le regard perdu à l'horizon, comme il se doit... ou du moins les yeux fixés sur une femme de chambre affairée autour du foyer. En Espagne, c'est Sara Montiel qui nous apprit à fumer pour ne pas consumer la vie trop vite... car la mélancolie, c'est bien cela, la vie que nous voyons se consumer. Mais il ne faut pas s'étonner que les fumeurs soient absents de cette exposition, étant donné que l'éternelle cigarette a été effacée de la main même de Sartre sur la dédicace du catalogue.

4 En Espagne, pour lutter contre la mélancolie, il ne nous restera bientôt plus que les émissions humoristiques à la télé (si nombreuses et amusantes, n'est-ce pas...) ou le Prozac. Ce sont les moyens les plus sains et les plus modernes. Personnellement, l'interdiction de fumer sur les lieux de travail ne me touche pas vraiment, car il y a longtemps déjà que j'ai pris soin de travailler chez moi. En outre, je suis tout à fait d'accord pour épargner cette gêne aux non-fumeurs avec lesquels je vis, mais je suis contre les mensonges révoltants. Or, contrairement à ce qu'on dit, fumer ne tue pas, même si fumer beaucoup n'est certainement pas bon pour la santé (je ne crois pas non plus que les œufs au bacon tuent, bien qu'en manger matin, midi et soir ne soit pas bénéfique pour le foie) et il doit y avoir autant de morts parmi les fumeurs passifs que parmi les personnes souffrant de la musique stridente que leur imposent des voisins sans-gêne. Mais en tout état de cause, indépendamment du préjudice pour autrui, je ne vois pas pourquoi le gouvernement devrait s'immiscer dans la régulation des vices. À moins qu'il partage l'avis du leader de la révolution iranienne, ayant récemment déclaré que "les droits de l'homme conduisent à l'immoralité». En ce qui concerne les mesures actuelles contre les fumeurs, la droite et la gauche seront cependant toujours d'accord. Les gouvernements de gauche évoquent les très importantes dépenses publiques consacrées aux victimes de l'abus de tabac. Mais, à bien y réfléchir, avec les taxes que les fumeurs paient sur le tabac, ils assument les frais d'une bonne part de la santé publique... La droite, pour sa part, ne peut pas s'empêcher d'interdire une source de plaisir, surtout quand il s'agit d'un plaisir populaire, à la portée de tout le monde. Si c'était au moins un plaisir exquis et minoritaire, comme manger du caviar ou posséder un yacht! Leur seul souci consiste à savoir si cette nouvelle inquisition nuira à la prospérité de certains commerces. 
Courageusement, la ministre espagnole de la Santé déclare aujourd'hui qu'une loi semblable contre l'alcool est à l'étude. La seule chose qui les arrête pour l'instant est que les recherches scientifiques appropriées, sur les effets nocifs de cette drogue pour l'être humain, n'ont pas encore été menées à bien. Mais il est difficile d'imaginer ce qu'une loi anti-fumeurs et une loi anti-buveurs pourraient avoir de commun, à moins de reconnaitre l'existence de buveurs passifs finissant par attraper une cirrhose par le simple fait qu'ils voient leurs proches soutirer le vin. Néanmoins, il doit sûrement y avoir déjà une statistique de l'OMS qui établit ce critère. Quant aux effets nocifs de l'alcool, je peux cette fois être utile à la ministre, car depuis de nombreuses années, je me livre à des expériences fréquentes, non dépourvues de risque personnel, avec cette substance, ou plutôt, avec des vins et des liqueurs, car l'alcool à proprement parler, je ne l'ai essayé que par voie externe. Je me mets entièrement à la disposition de la ministre. En tout cas, il est évident à première vue qu'il ne doit pas s'agir d'un poison très puissant, car on n'a jamais interdit d'absorber des substances empoisonnantes. Il n'y a, en effet, pas de loi défendant de boire de la lessive ou de l'acide prussique, par exemple. Autrement dit, seul ce que beaucoup de gens désirent peut être décrété indésirable et non pas ce que personne ne veut. On condamnera, une nouvelle fois, l'usage au nom de l'abus, comme pour le tabac. D'ailleurs, puisqu'on parle de tous les morts dont la drogue est responsable, j'en profite ici pour féliciter le Dr Albert Hoffman pour ses cent premières années, qu'il porte très bien... Mais il est choquant de voir que les défenseurs de la diversité culturelle observent un répit dans ces cas-là : n'y aurait-il pas, par hasard, de culture du tabac ou du vin? Manifestement, les aspirations multiculturelles trouvent ici leur limite. La civilisation de la santé ne crée pas d'alliances, uniquement des prisonniers ou des esclaves...

6 Le pire, cependant, à mon vicieux avis, est le petit air de vertu qui entoure la proclamation joyeuse de telles mesures. Comme l'a remarqué Chesterton: "s'il y a quelque chose de pire que l'affaiblissement moderne des grands principes moraux, c'est le renforcement des petits principes moraux». En effet, notre société se veut toujours plus vertueuse, mais avec un v de plus en plus minuscule. En français, l'expression " petite vertu » désigne les attitudes aimables de dames délicieuses : rien à voir, bien entendu, avec nos ministres. Sur ce, je m'en retourne, sans remède, à la mélancolie...

7 Traduit de l'espagnol par Aurélien Talbot.

\section{RÉSUMÉS}

"Avec un v minuscule». L'apologie de la vertu « avec un v minuscule », caractéristique de nos sociétés occidentales contemporaines, nous livre sans défense à la mélancolie...

"With a small v". The apology of virtue, with a small v, a characteristic of our present occidental civilisation, leaves unarmed in face of melancholy.

"Con uve minúscula...". La apología de la virtud « con uve minúscula », que es característica de nuestras sociedades occidentales, nos entrega indefensos a la melancolía... 
"Die Tugend, klein geschrieben". Die Verherrlichung der Tugend, so charakterisch für unsere westlichen zeitgenössischen Gesellschaften, liefere uns wehrlos der Melancholie aus.

\section{AUTEUR}

\section{FERNANDO SAVATER}

Fernando Savater est professeur de philosophie à Madrid. Il est l'auteur de plus de cinquante ouvrages, essentiellement de sciences humaines. Il collabore régulièrement au quotidien El País. Parmi ses œuvres traduites en français, mentionnons Pour l'éducation (Payot \& Rivages, 2000), Dictionnaire philosophique personnel (Grasset-Mollat, 1999) ou encore Éthique à l'usage de mon fils (Seuil, 1994). 